\title{
2018 Year in Review: Noninvasive Respiratory Support
}

\author{
John D Davies
}

\author{
Introduction \\ Noninvasive Respiratory Support for the Subject Who Is \\ Immunocompromised \\ Use of NIV for Preoxygenation Before Endotracheal Intubation \\ Noninvasive Respiratory Support in Subjects With Hypoxemic \\ Respiratory Failure \\ Noninvasive Respiratory Support and Weaning \\ Noninvasive Respiratory Support in Subjects Who Are Critically Ill \\ Noninvasive Respiratory Support in Subjects With ARDS \\ Noninvasive Respiratory Support in the Neonatal and Pediatric Population \\ Summary
}

\begin{abstract}
Noninvasive respiratory support refers to strategies aimed at providing oxygenation and/or ventilation without the use of an artificial airway. These strategies include the use of standard oxygen delivery devices (face masks, low-flow nasal cannulas), noninvasive ventilation, and high-flow nasal cannula. Considerable interest has been generated recently as to which therapy provides the optimum noninvasive support. This review examined the important literature related to noninvasive respiratory support published in 2018. Key words: noninvasive ventilation; standard oxygen therapy; high-flow nasal cannula; continuous positive airway pressure; hypercarbic respiratory failure; hypoxemic respiratory failure; immunocompromised; preoxygenation; weaning; ARDS; bronchiolitis; transport. [Respir Care 2019;64(9):1139-1145. (C) 2019 Daedalus Enterprises]
\end{abstract}

\section{Introduction}

Noninvasive respiratory support encompasses strategies such as standard oxygen delivery, noninvasive ventilation (NIV), CPAP, and high-flow nasal cannula (HFNC) ther-

\footnotetext{
Mr Davies is affiliated with Duke University Health System, Durham, North Carolina.

Mr Davies presented a version of this paper at the Year in Review of the AARC Congress 2018, held December 4-7, 2018, in Las Vegas, Nevada.

Mr Davies discloses a relationship with Teleflex Medical.
}

Correspondence: John D Davies MA RRT FAARC, Respiratory Care Services, Duke University Hospital, Box 3911, Durham, North Carolina 27710. E-mail:john.davies@duke.edu.

DOI: $10.4187 /$ respcare. 07170 apy. Standard oxygen delivery uses different types of interfaces to provide oxygen delivery. The most common interfaces are: simple oxygen masks, nasal cannulas, partial rebreather masks, non-rebreather masks, air-entrainment masks, face tents, and tracheostomy collars. NIV and CPAP are also used with a variety of interfaces. Among the more common interfaces are the nasal mask, oronasal mask, total face mask, and nasal pillows. HFNC is the new kid on the block and has the capability of delivering high $\mathrm{F}_{\mathrm{IO}_{2}}$ in conjunction with higher flows than the standard nasal cannula. This review examined some of the recent literature that pertains to noninvasive respiratory support over the past year. Clinical scenarios in which noninvasive support have been studied recently include the following: in subjects who are immunocompromised with respiratory failure, for preoxygenation prior to intubation, in subjects with hypoxemic respiratory failure, weaning patients from 
mechanical ventilation, subjects who are critically ill, in subjects with ARDS and in the pediatric and neonatal population.

\section{Noninvasive Respiratory Support for the Subject Who Is Immunocompromised}

Acute respiratory failure is the most common reason that patients who are immunocompromised are admitted to the ICU. ${ }^{1}$ Already at risk as a consequence of immunocompromise, these patients who require intubation and mechanical ventilation have a greater mortality risk. ${ }^{2}$ Therefore, noninvasive strategies to avoid this scenario are highly prized. The question is, do they work?

Azoulay et $\mathrm{al}^{3}$ attempted to answer this question by performing a randomized clinical trial examining the use of high flow nasal oxygen therapy as compared to the use of standard oxygen therapy in immunocompromised patients with acute respiratory failure. The study was conducted in 32 ICUs in France. They recruited 388 subjects with hypoxemic respiratory failure defined as a $\mathrm{P}_{\mathrm{aO}_{2}}$ of $<60 \mathrm{~mm} \mathrm{Hg}$ or oxygen saturation by a pulse oximetry of $<90 \%$ while breathing room air, or tachypnea ( $>30$ breaths/min) and the need for oxygen flow $>6 \mathrm{~L} / \mathrm{min}$ by cannula. ${ }^{3}$ The primary cause of immunosuppression in these subjects stemmed from the use of long-term or high-dose steroids, the use of immunosuppressant drugs, solid organ transplantation, chemotherapy within the past 5 years for a solid tumor, hematologic malignancy, or primary immune deficiency. ${ }^{3}$

The subjects were randomized to receive either the intervention (high-flow oxygen therapy) or standard oxygen therapy during their ICU stay; the intervention group received HFNC with initial flows of $50 \mathrm{~L} / \mathrm{min}$ and $100 \%$ oxygen. ${ }^{3}$ The flows and oxygen concentrations were then adjusted to maintain a $\mathrm{S}_{\mathrm{pO}_{2}}>95 \%$, with a maximum flow of $60 \mathrm{~L} / \mathrm{min} .{ }^{3}$ The control group received oxygen via any device or combination of devices used for standard care. As with the intervention group, the $\mathrm{S}_{\mathrm{pO}_{2}}$ target was $>95 \%$. $^{3}$ The primary outcome was 28 -d mortality, which was not significantly different between the groups $(P=.94) .{ }^{3}$ For the group that received HFNC, $35.6 \%$ of the subjects (138 of 388) had died by day 28 . The group that received standard-care oxygen had a $36.1 \%$ 28-d mortality (140 of 388). There was also no difference in the need for intubation, ICU-acquired infection, comfort, dyspnea, or ICU stay. There was an important limitation to this study in that all the participating centers were located in France, so one needs to take caution in assuming that these results apply worldwide.

Sklar et $\mathrm{al}^{4}$ performed a systematic review and metaanalysis of the impact of HFNC in subjects who were immunocompromised and critically ill. They included 13 studies with a total of 1,956 subjects. The types of studies included 8 retrospective cohort studies (532 subjects); 1 prospective, observational study (859 subjects); and 4 randomized controlled studies (565 subjects). ${ }^{4}$ The main causes of immunosuppression were related to an oncologic diagnosis or solid organ transplantation. The primary outcome investigated was mortality, with a secondary outcome of the rate of mechanical ventilation for HFNC compared with NIV and/or standard oxygen therapy. ${ }^{4}$ Three of the studies compared HFNC with both NIV and standard oxygen therapy, 3 compared HFNC with NIV alone, 3 compared HFNC with standard oxygen therapy, and 4 studies had no control group.

The investigators found a mortality benefit for HFNC over all conventional therapies combined (both NIV and standard oxygen therapy). When HFNC was compared with NIV and standard oxygen therapy separately, there was a mortality benefit for HFNC over NIV $(P=.04)$ but not over standard oxygen therapy $(P=.11) .{ }^{4}$ There were no significant differences in the rate of invasive ventilation for HFNC compared with NIV or standard oxygen therapy. A number of limitations were identified: (1) there was a small number of high-quality studies, and (2) there was heterogeneity with respect to the underlying cause of immunosuppression, the definition of respiratory failure, the types of oxygen strategies used, and the criteria for intubation. ${ }^{4}$ The investigators concluded that HFNC has theoretical promise but, due to the paucity of high-quality data, it cannot, at this point, be considered evidence-based therapy. ${ }^{4}$

These studies represent the conflicting evidence as to whether noninvasive respiratory support is clinically beneficial for patients with respiratory failure and who are immunocompromised. Azoulay et $\mathrm{al}^{3}$ compared HFNC with standard oxygen therapy and found no benefit. However, Sklar et $\mathrm{al}^{4}$ indicated that some benefit may exist for noninvasive respiratory support in patients who are immunocompromised. Clearly, much more research is needed in this area.

\section{Use of NIV for Preoxygenation Before Endotracheal Intubation}

Preoxygenation is a vital step in the endotracheal intubation process to avoid or delay hypoxemia during the procedure. NIV has the advantage over standard oxygen delivery via face mask in that, while being able to also deliver high-oxygen concentrations, NIV can unload respiratory muscles and potentially increase lung volumes.

Baillard et $\mathrm{al}^{5}$ undertook a study to see if NIV was more effective than face-mask oxygen delivery before intubation with respect to organ dysfunction. This group performed a multi center ( 6 centers) randomized open-label trial that compared NIV set at an $\mathrm{F}_{\mathrm{IO}_{2}}$ of 1.0 for $3 \mathrm{~min}$ before intubation versus face-mask oxygen (non-rebreath- 
ing bag-valve-mask) delivery with an $\mathrm{F}_{\mathrm{IO}_{2}}$ of $1.0(N=201) .^{5}$ For the NIV group, pressure support was the ventilatory mode used and the tidal volume was maintained at $6-8 \mathrm{~mL} / \mathrm{kg}$ predicted body weight. PEEP of $5 \mathrm{~cm} \mathrm{H}_{2} \mathrm{O}$ was also applied. After intubation, each subject was maintained on a mechanical ventilator at the following settings: tidal volume $6-8 \mathrm{~mL} / \mathrm{kg}$ predicted body weight, frequency 20 breaths/min, PEEP $5 \mathrm{~cm} \mathrm{H}_{2} \mathrm{O}$, and $\mathrm{F}_{\mathrm{IO}_{2}}$ 1.0.5 The primary end point was the maximum Sequential Organ Failure Assessment score in the 7-d period after intubation.

They reported no difference between the groups in terms of maximum Sequential Organ Failure Assessment score in the 7-d period after intubation; they also failed to demonstrate any difference in the duration of mechanical ventilation or ICU stay. ${ }^{5}$ However, one important finding was detected. In subjects who were receiving NIV before randomization for the study, there was a significant increase in the frequency of adverse events in those who went into the face-mask group $(P=.006) .{ }^{5}$ The investigators concluded that, although there was no difference in terms of the preoxygenation method, the subjects who were receiving NIV before preoxygenation should continue on NIV during preoxygenation and not be switched to bag-valvemask before intubation. ${ }^{5}$ It could be that a lack of fine ventilatory control (inspiratory pressure and tidal volume) when using a bag-valve-mask compared with NIV for preoxygenation results in excessive inspiratory pressures and tidal volumes that may be harmful. Also, in many instances, preoxygenation with a bag-valve-mask setup is done without the use of a PEEP valve. This lack of PEEP may lead to a more rapid loss of recruitment, whereas, with NIV use before and during preoxygenation, PEEP (and recruitment) is maintained.

\section{Noninvasive Respiratory Support in Subjects With Hypoxemic Respiratory Failure}

In a previous large multi center randomized controlled trial of 310 subjects with acute hypoxemic respiratory failure, HFNC was shown to have an intubation and mortality benefit over both NIV and standard oxygen therapy. ${ }^{6}$ Frat et $\mathrm{al}^{7}$ followed that study with a post hoc analysis that attempted to identify early respiratory-related factors associated with intubation and, in the ICU, in subjects with hypoxemic respiratory failure being treated with noninvasive respiratory support. This post hoc analysis examined subjects who were receiving standard oxygen therapy, NIV, and HFNC. ${ }^{7}$

Standard oxygen therapy consisted of a non-rebreathing mask with an oxygen flow of at least $10 \mathrm{~L} / \mathrm{min} .{ }^{7}$ NIV was delivered via face mask for a duration of $\geq 8 \mathrm{~h}$ per day, with HFNC being applied when the subjects were off NIV. Pressure support was used during NIV, and the target tidal volume was $7-10 \mathrm{~mL} / \mathrm{kg}$ of predicted body weight, and
PEEP was initially set at $5 \mathrm{~cm} \mathrm{H}_{2} \mathrm{O} .^{7} \mathrm{HFNC}$ was set at $50 \mathrm{~L} / \mathrm{min}$ and, in all 3 groups, the target $\mathrm{S}_{\mathrm{pO}_{2}}$ was $>92 \%$. The investigators reported that, in the standard oxygen group, a frequency of $>30$ breaths/min was associated with intubation $1 \mathrm{~h}$ after initiation. ${ }^{7}$ In the NIV group, a high tidal volume ( $>9 \mathrm{~mL} / \mathrm{kg}$ predicted body weight) after $1 \mathrm{~h}$ was associated with intubation; no respiratory variable was associated with intubation in the HFNC group. ${ }^{7}$

\section{Noninvasive Respiratory Support and Weaning}

Recent guidelines support the use of NIV to facilitate weaning from mechanical ventilation in patients with hypercarbic respiratory failure. ${ }^{8}$ However, it is unknown whether NIV can have a weaning benefit for the general population. Perkins et $\mathrm{al}^{9}$ performed a multi center randomized controlled clinical trial to determine if there might be benefit to using NIV to facilitate weaning in a heterogeneous population of subjects who were classified as difficult to wean. The trial took place in 41 adult ICUs in the United Kingdom and included 364 subjects. ${ }^{9}$ Subjects were enrolled if they were on invasive mechanical ventilation for $>48 \mathrm{~h}$ and were deemed ready to wean (ready to undergo a spontaneous breathing trial). Patients for whom their first spontaneous breathing trial failed were considered difficult to wean. The subjects were randomized to the intervention group (which consisted of extubation to NIV) or the control group (protocolized sequential pressure support weaning with spontaneous breathing trials). ${ }^{9}$ The main admission diagnoses included subjects with pneumonia or respiratory infection, postsurgery respiratory failure, and cardiac-related and non-respiratory infections.

There were 7 subjects in each of the intervention and control groups who had a COPD diagnosis. ${ }^{9}$ In the NIV group, once the treating clinician deemed the subject ready to wean, he or she was extubated directly to NIV. The inspiratory and expiratory pressure levels were adjusted to match the pre-extubation settings. The primary outcome was the time from randomization to liberation from the ventilator (either invasive or noninvasive) for $>48 \mathrm{~h}$. The main finding of this study was that there was no difference in the time of randomization to successful liberation from mechanical ventilation. As would be expected, the NIV group required less invasive mechanical ventilation time and this group required more reintubations (37\% vs $28 \%$ in the control group). The higher re-intubation rate for the NIV group is a concern for the associated increase in mortality but, in this study, the survival rates were not significantly different. Clearly, there are specific patients who would benefit from early extubation (those with COPD),${ }^{8}$ but Perkins et $\mathrm{al}^{9}$ showed why we should be careful when using this practice in a heterogeneous ICU population. 


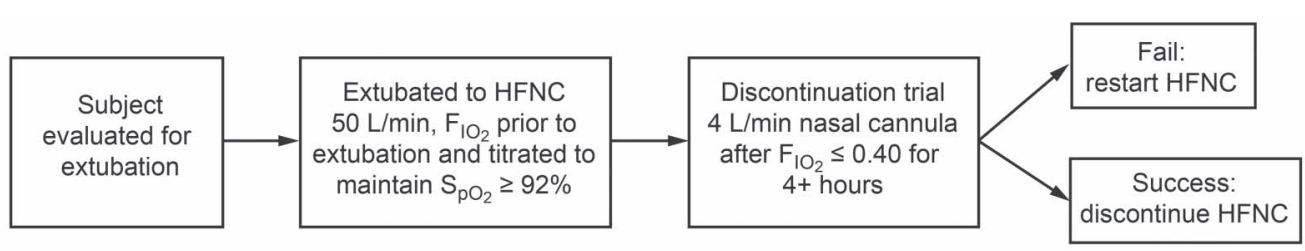

Fig. 1. Study protocol for cohort 1. From Reference 10.

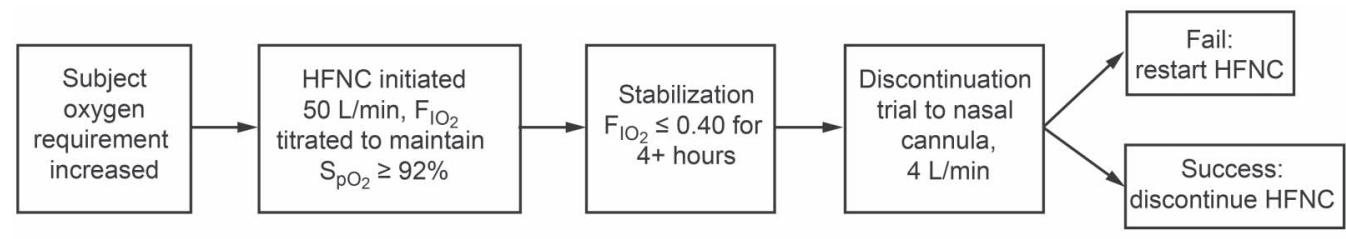

Fig. 2. Study protocol for cohort 2. From Reference 10.

\section{Noninvasive Respiratory Support in Subjects Who Are Critically Ill}

HFNC use has become increasingly popular in the populations of patients who are critically ill because clinicians can now deliver high $\mathrm{F}_{\mathrm{IO}_{2}}$ at a high flow through a nasal cannula. It carries the advantage of maintaining patient comfort and adequate humidification, and it avoids bypassing the upper-airway defense mechanisms. Lamb et al ${ }^{10}$ examined the use of HFNC in subjects who were critically ill by separating them into 2 cohorts: (1) subjects who were extubated directly to HFNC after receiving mechanical ventilation for $\geq 24 \mathrm{~h}$, and (2) subjects who were not intubated and who required an escalation of respiratory support. Cohort 1 consisted of 88 subjects who were placed directly on HFNC when extubated at $50 \mathrm{~L} / \mathrm{min}$, and the last documented $\mathrm{F}_{\mathrm{IO}_{2}}$ setting on the ventilator and adjustments were made, if needed, to keep the $\mathrm{S}_{\mathrm{pO}_{2}} \geq 92 \% .^{10}$ When the $\mathrm{F}_{\mathrm{IO}_{2}}$ was $\leq 0.40$ for 4 consecutive hours, the subjects were given a trial of conventional nasal cannula at $4 \mathrm{~L} / \mathrm{min}$. If the trial failed, then they were placed back on HFNC at the previous settings (Fig. 1). ${ }^{10}$

Lamb et $\mathrm{al}^{10}$ used a similar-size control group that consisted of retrospectively identified subjects. In this group, postextubation respiratory management was at the discretion of the clinical team. Cohort 2 consisted of 83 subjects who required $>4 \mathrm{~L} / \mathrm{min}$ nasal cannula or an $\mathrm{F}_{\mathrm{IO}_{2}}$ of $\geq 0.36$ to maintain the $\mathrm{S}_{\mathrm{pO}_{2}} \geq 92 \% .{ }^{10}$ The HFNC protocol was managed in the same manner as cohort 1 in that the HFNC was started at $50 \mathrm{~L} / \mathrm{min}$ and the $\mathrm{F}_{\mathrm{IO}_{2}}$ was adjusted to keep the $\mathrm{S}_{\mathrm{pO}_{2}}$ of $\geq 92 \%$ (Fig. 2). ${ }^{10}$ The main findings of this study were the following: (1) intubation rates were not different between the pre-protocol and the protocol groups for both cohorts; (2) in cohort 1, the incidence of Gramnegative pneumonia was lower in the protocol group $(P=.001) ;(3)$ although not statistically significant, there was a slight mortality benefit trend in the protocol in cohort 1 of $9 \%$ versus $13 \%$ ( $P=.63)$, (4) bronchodilator use decreased in the protocol groups in both cohorts, with the decrease in cohort 1 being statistically significant $(P=.008) ;(5)$ in cohort 2 , the protocol group had a significantly lower ICU and hospital stay $(P=.03$ and $P=.007$, respectively); and (6) when HFNC failed in the subjects, the time to intubation was much shorter than in the control groups. ${ }^{10}$ The decrease in the incidence of Gramnegative pneumonia could represent improved mucociliary clearance due to the increase in humidity delivery with HFNC as opposed to conventional oxygen therapy. The decrease in bronchodilator use and rapid intubation times with HFNC failure could reflect the value of standardized protocols and the increased monitoring frequency. The study is limited due to the retrospective nature of the control groups, and, therefore, the results must be taken in that context. However, in this study, the use of HFNC yielded some promising results.

One of the common concerns with NIV is that, despite technological advances that have produced more comfortable interfaces, there are still times when the interface may be uncomfortable for patients. These patients may require breaks from NIV to relieve the pressure applied to the face from the mask. They may also need breaks from NIV to eat and to communicate with family members. In these circumstances, during breaks from NIV, patients are usually placed on some sort of standard oxygen device, usually a nasal cannula or air-entrainment mask. Most of the previous interest in HFNC is as a replacement for NIV not in conjunction with NIV. HFNC may offer an attractive alternative to standard oxygen therapy to provide respiratory support during NIV breaks.

Spoletini et al ${ }^{11}$ investigated this potential alternative through an unblinded multi center randomized parallel controlled trial. They randomized subjects to receive either 
standard oxygen therapy or HFNC during breaks from NIV. Forty-seven adult subjects were recruited if their anticipated time on NIV was $>24 \mathrm{~h}$ and if they had acute or acute-on-chronic hypercarbic respiratory failure or acute hypoxemic respiratory failure. On NIV, the subjects received pressure-support ventilation with tidal volumes of $6-8 \mathrm{~mL} / \mathrm{kg}$ predicted body weight and frequency $\leq 24$ breaths/min, and the $\mathrm{S}_{\mathrm{pO}_{2}}$ was kept at $88-92 \%$ for those with hypercapnic respiratory failure and $\geq 92 \%$ for subjects with hypoxemic respiratory failure. ${ }^{11}$ The subjects in the standard oxygen therapy group were set up with either a nasal cannula, air-entrainment mask, or nonrebreathing mask during the NIV breaks.

In the intervention group, the subjects were placed on an HFNC with an initial flow of $35 \mathrm{~L} / \mathrm{min}$ and then both the flow and $\mathrm{F}_{\mathrm{IO}_{2}}$ were adjusted to keep the $\mathrm{S}_{\mathrm{pO}_{2}}$ in the defined ranges. ${ }^{11}$ NIV was restarted if the subjects experienced uncontrolled dyspnea, increased breathing frequency, increased heart rate (15\% above baseline), systolic blood pressure that either increased or decreased by $20 \%$, a drop in the $\mathrm{S}_{\mathrm{pO}_{2}}$ that did not respond to increased settings, or a request by either the subject or the treating clinician to restart NIV. ${ }^{11}$ The primary outcome investigated the total time spent on NIV. The investigators found that there was no difference in the amount of time spent on NIV nor was there a difference in the total time spent on breaks from NIV. ${ }^{11}$ Where HFNC seemed to have an advantage was in the area of comfort. Dyspnea scores trended lower in the HFNC, and, not surprisingly, the patients had less trouble eating. Although this study did not demonstrate a tangible benefit for the use of HFNC, it does highlight the importance of how best clinicians should manage a patient's respiratory support during breaks from NIV.

\section{Noninvasive Respiratory Support in Subjects With ARDS}

Surviving the initial insult from ARDS and its associated therapies is an important first step in recovery. However, there can be a long recovery time that could include neuromuscular weakness and functional impairment due to the use of neuromuscular blockade and extended time on mechanical ventilation and without physical activity. In a previous randomized controlled trial, Patel et al ${ }^{12}$ showed that using a helmet to deliver NIV could reduce intubation rates (by $40 \%$ ) when compared with face-mask NIV. Based on this previous work, the group performed a 1-year follow-up study of the same subjects. Patel et al ${ }^{13}$ evaluated subjects who survived to hospital discharge and had a functional interdependence assessment (degree of independence in performing daily living activities and ambulation) done at discharge. One year later, these subjects were interviewed by telephone. Functional independence and survival were assessed. The results were that $48 \%$ of the subjects who received NIV via the helmet returned home on hospital discharge compared with only $21 \%$ of the face-mask group $(P<.001) .{ }^{13}$ Half of the subjects in the helmet group $(50 \%)$ were considered functionally independent versus $15 \%$ in the face-mask group $(P=.001)$, and survival rates were better as well in the helmet group, $57 \%$ versus $31 \%(P=.007) .{ }^{13}$ NIV delivered via helmet is an intriguing interface but its use is mainly limited to Europe. The use of helmet NIV in ARDS merits further investigation.

\section{Noninvasive Respiratory Support in the Neonatal and Pediatric Population}

Noninvasive respiratory support is gaining popularity in the neonatal and pediatric populations. In this section, we examined its use in bronchiolitis, low birthweight infants, and during patient transport. Bronchiolitis is a lower-airway disease caused by respiratory viruses and usually results in small-airway inflammation that leads to hypoxia and hypercarbia. The most recent guidelines for management of bronchiolitis mainly call for supportive care, which includes oxygen therapy and respiratory support. ${ }^{14}$ In most cases, the therapy given for bronchiolitis is oxygen via a standard nasal cannula. Franklin et al ${ }^{15}$ conducted a large multi center randomized trial of 1,472 infants to investigate any potential benefit that HFNC may have over standard oxygen therapy. Infants $<1$ y old who had clinical signs of bronchiolitis that required oxygen therapy to maintain an oxygen saturation of $\geq 92 \%$ were included. ${ }^{15}$ The HFNC group received heated and humidified high-flow oxygen at $2 \mathrm{~L} / \mathrm{kg}$ of body weight per minute. ${ }^{15}$ The $\mathrm{F}_{\mathrm{IO}_{2}}$ was adjusted to maintain oxygen saturations of $92-98 \% .{ }^{25}$ HFNC was terminated when the subject tolerated $4 \mathrm{~h}$ of $\mathrm{HFNC}$ at an $\mathrm{F}_{\mathrm{IO}_{2}}$ of 0.21 .15

The standard oxygen group received oxygen through a conventional nasal cannula up to a maximum of $2 \mathrm{~L} / \mathrm{min} .{ }^{15}$ The primary outcome of the study was treatment failure defined as escalation of care. There were 4 clinical criteria that, when at least 3 were met, resulted in escalation of care (treatment failure). The criteria were the following (1) unchanged or increased heart rate, a decrease of $>5$ beats $/ \mathrm{min}$ or into the normal range was classified as success; (2) frequency remained unchanged or increased, a decrease of $>5$ breaths/min or into the normal range was classified as success; (3) $\mathrm{F}_{\mathrm{IO}_{2}}>0.40$ in the HFNC group or oxygen requirement $>2 \mathrm{~L} / \mathrm{min}$ in the control group; and (4) the hospital internal early warning tool triggered a medical review and escalation of care. ${ }^{15}$ This early warning tool consisted of a number of physiologic and clinical variables that were automatically monitored. The results of the study were that 87 of 739 in the HFNC group (12\%) required escalation of care that resulted in treatment failure, whereas 167 of 733 in the standard oxygen group (23\%) required 
escalation of care $(P<.001) .{ }^{15}$ There was also a clause in the study in which the clinical team could decide to escalate care (treatment failure) even if the patient did not meet at least 3 of the 4 escalation of care criteria. ${ }^{15}$ In this group, 53 of 254 in the HFNC group (7\%) required escalation of care, whereas that number for the standard oxygen group was 11 of $254(16 \%)(P<.001) .{ }^{15}$ The parents of all of the infants who had treatment failure in the standard oxygen group were offered the option of HFNC as escalation of care and accepted. Of these, 102 of 167 subjects (61\%) responded positively to HFNC, whereas, in 65 of 167 subjects (39\%), HFNC was not effective. ${ }^{15}$ There was no difference in adverse events, ICU or hospital length of stay, or duration of oxygen therapy. This study suggests that HFNC for patients with bronchiolitis and the need for oxygen therapy may perceive benefit without increased safety risk.

Drescher and Hughes ${ }^{16}$ examined the RAM cannula (Neotech, Valencia, CA), which, off-label, can deliver nasal CPAP and bi-level nasal NIV. They set out to determine if the RAM cannula can improve clinical outcomes over conventional devices in 72 low birthweight infants. The group conducted a prospective observational study that compared the RAM cannula with an historical control group in infants $<29$ weeks of gestational age and who weighed $<1,500 \mathrm{~g}$. The invention group included the RAM cannula in conjunction with HFNC and the RAM cannula in conjunction with NIV. In both groups, CPAP was usually the primary noninvasive mode used, starting at $5 \mathrm{~cm} \mathrm{H} \mathrm{H}_{2} \mathrm{O}$. Bi-level NIV was typically used if CPAP failed before intubation. HFNC was used as a transition phase from CPAP to a standard nasal cannula. The findings were that the RAM cannula group had a significantly lower number of days when (1) on CPAP, bi-level NIV, or HFNC ( $38 \mathrm{~d}$ in the RAM cannula group vs $47 \mathrm{~d}$ in the control group; $P=.02$ ); and (2) on any respiratory support ( 57 vs $74 \mathrm{~d} ; P=.009$ ). One other important finding is that skin or mucosal breakdown was significantly lower in the RAM cannula group (53\% of infants in the control group and $8 \%$ in the RAM cannula group $P<.001$ ). These results need to be taken with a fair degree of caution. It was a relatively small study, and it was observational in nature, with a retrospective control group as opposed to a randomized controlled trial.

The final study in pediatrics relates to using NIV during neonatal and pediatric transport. Up to this point, research that relates to NIV in children during transport is scarce. NIV during transport has considerable appeal, especially if it acts to avoid intubation, which might otherwise facilitate safe travel. Cheema et $\mathrm{al}^{17}$ attempted to explore the literature through a systematic review that relates to the use of NIV during pediatric and neonatal transports. In particular, the group looked at intubation rates during or within $24 \mathrm{~h}$ of transfer to the health-care facility, escalation to invasive mechanical ventilation, and the incidence of adverse events during the transport. Secondary outcomes examined were clinical parameters, including heart, breathing frequency, oxygen saturation, and work of breathing. The group was able to identify 8 studies, which included 858 pediatric subjects transported while on NIV; 708 subjects on CPAP, and, surprisingly 150 on HFNC ( 1 study). ${ }^{17}$

Unfortunately, all of the above mentioned studies were observational in nature, which highlights the paucity of evidence for pediatric or neonatal NIV, CPAP, and HFNC during transport. Intubation was only performed in 3 of the 858 subjects; 1 of 708 received CPAP, and none in subjects who received HFNC during transport. Escalation to CPAP or bi-level NIV occurred in 2 subjects who were receiving HFNC. Intubation within $24 \mathrm{~h}$ of the transfer occurred in 60 of 500 subjects (12\%) who received CPAP and 3 of 150 subjects who received HFNC. Escalation to either CPAP or bi-level NIV within the first $24 \mathrm{~h}$ after transport happened in 9 of 150 of the subjects $(6 \%)$ who received HFNC. There were a few adverse events reported, but the actual reporting was incomplete in most of the studies. However, the adverse events that were reported included bag-valve-mask ventilation $(1 \%)$, desaturation $(2 \%)$, and apnea (4\%). Reporting was also unreliable and incomplete for secondary outcomes, thus, no meaningful conclusions can be made. Due to the heterogeneity of the study population, no definitive conclusions could be drawn; however, the analysis of the results suggest that the use of noninvasive respiratory support other than standard oxygen therapy may be safe in the pediatric and neonatal populations during transport. Much more research is desperately needed in this area.

\section{Summary}

This review represents a snapshot of the literature published in 2018 related to noninvasive respiratory support. However, it does provide the reader with an idea of how the interest in HFNC is expanding to include new potential indications. Intuitively, it seems that HFNC should be of benefit for the patients who are immunocompromised but the research has not definitively supported this notion, with conflicting data being presented. The interest in NIV also continues to grow as it has for the past 2 decades. One study indicates that NIV may have a role in preoxygenation support in certain patients. ${ }^{5}$ Both HFNC and NIV continue to be explored in patients who are critically ill and in patients with hypoxemic respiratory failure. HFNC and NIV are generating a lot of interest in the pediatric and neonatal populations as well. Standard oxygen therapy remains the initial default therapy for most disease conditions and respiratory-related ailments. 


\section{NONINVASIVE RESPIRATORY SUPPORT}

\section{REFERENCES}

1. Mokart D, Pastores SM, Darmon M. Has survival increased in cancer patients admitted to the ICU? Yes. Intensive Care Med 2014;40(10): 1570-1572.

2. Azoulay E, Mokart D, Pène F, Lambert J, Kouatchet A, Mayaux J, et al. Outcomes of critically ill patients with hematologic malignancies: prospective multicenter data from France and Belgium-a groupe de recherché respiratoire en reanimation onco-hematologique study. J Clin Oncol 2013;31(22):2810-2818.

3. Azoulay E, Lemiale V, Mokart D, Nseir S, Argaud L, Pène F, et al. Effect of high-flow nasal oxygen vs standard oxygen on 28-day mortality in immunocompromised patients with acute respiratory failure. The HIGH randomized clinical trial. JAMA 2018;320(20):2099-2107.

4. Sklar MC, Mohammed A, Orchanian-Cheff A, Del Sorbo L, Mehta $\mathrm{S}$, Munshi L. The impact of high-flow nasal oxygen in the immunocompromised critically ill: a systematic review and meta-analysis. Respir Care 2018;63(12):1555-1566.

5. Baillard C, Prat G, Jung B, Futier E, Lefrant JY, Vincent F, et al. Effect of preoxygenation using non-invasive ventilation before intubation on subsequent organ failures in hypoxemic patients: a randomized clinical trial. Br J Anaesth 2018;120(2):361-367.

6. Frat JP, Thille AW, Mercat A, Girault C, Ragot S, Perbet S, et al.; FLORALI Study Group, REVA Network. High-flow oxygen through nasal cannula in acute hypoxemic respiratory failure. N Engl J Med 2015;372(23):2185-2196

7. Frat JP, Ragot S, Coudroy R, Constantin JM, Girault C, Prat G, et al.; REVA network. Predictors of intubation in patients with acute hypoxemic respiratory failure treated with a noninvasive oxygenation strategy. Crit Care Med 2018;46(2):208-215.

8. Rochwerg B, Brochard L, Elliott MW, Hess DR, Hill NS, Nava S, et al.; Navalesi P Members of the Steering Committee, Raoof S Members of the Task Force. Official ERS/ATS clinical practice guidelines: noninvasive ventilation for acute respiratory failure. Eur Respir J 2017;50(2). pii: 1602426.
9. Perkins GD, Mistry D, Gates S, Gao F, Snelson C, Hart N, et al.; Breathe Collaborators. Effect of protocolized weaning with early extubation to noninvasive ventilation vs invasive weaning on time to liberation from mechanical ventilation among patients with respiratory failure: the Breathe Randomized Clinical Trial. JAMA 2018; 320(18):1881-1888.

10. Lamb KD, Spilman SK, Oetting TW, Jackson JA, Trump MW, Sahr SM. Proactive use of high-flow nasal cannula with critically ill subjects. Respir Care 2018;63(3):259-266.

11. Spoletini G, Mega C, Pisani L, Alotaibi M, Khoja A, Price LL, et al. High-flow nasal therapy vs standard oxygen during breaks off noninvasive ventilation for acute respiratory failure: a pilot randomized controlled trial. J Crit Care 2018;48:418-425.

12. Patel BK, Wolfe KS, Pohlman AS, Hall JB, Kress JP. Effect of noninvasive ventilation delivered by helmet vs face mask on the rate of endotracheal intubation in patients with acute respiratory distress syndrome: a randomized clinical trial. JAMA 2016;315(22):24352441.

13. Patel BK, Wolfe KS, MacKenzie EL, Salem D, Esbrook CL, Pawlik $\mathrm{AJ}$, et al. One-year outcomes in patients with acute respiratory distress syndrome enrolled in a randomized clinical trial of helmet versus facemask noninvasive ventilation. Crit Care Med 2018;46(7): 1078-1084.

14. Zorc JJ, Hall CB. Bronchiolitis: recent evidence on diagnosis and management. Pediatrics 2010;125(2):342-349.

15. Franklin D, Babl FE, Schlapbach LJ, Oakley E, Craig S, Neutze J, et al. A randomized trial of high-flow oxygen therapy in infants with bronchiolitis. N Engl J Med 2018;378(12):1121-1131.

16. Drescher GS, Hughes CW. Comparison of interfaces for the delivery of noninvasive respiratory support to low birthweight infants. Respir Care 2018;63(10):1197-1206.

17. Cheema B, Welzel T, Rossouw B. Noninvasive ventilation during pediatric and neonatal critical care transport: a systematic review. Pediatr Crit Care Med 2019;20(1):9-18. 